\title{
Influence de la surface corporelle sur les indices de voltage ventriculaire gauche chez des sédentaires sains
}

\author{
Appolinaire KOUAME BROU ${ }^{1,2^{*}}$, Edwige BALAYSSAC-SIRANSY ${ }^{1,2}$, \\ Kossiwa DJAHA-TOUMATA ${ }^{2}$, Michel KOUASSI BROU ${ }^{1,3}$, Liliane KONDO $^{1}$, \\ Joel BOKA KOTCHI ${ }^{1}$, Audrey ASSI ${ }^{1}$, Paule-Denise YAPO $^{1}$, Soualiho OUATTARA ${ }^{1}$, \\ Cyrille DAH ${ }^{1,3}$ et Pascal BOGUI ${ }^{1,2}$ \\ ${ }^{1}$ Laboratoire de physiologie et explorations fonctionnelles de l'UFR Sciences Médicales d'Abidjan, \\ Côte d'ivoire. \\ ${ }^{2}$ Service des explorations fonctionnelles et endoscopiques du CHU de Yopougon, Abidjan, Côte d'Ivoire. \\ ${ }^{3}$ Service des explorations fonctionnelles du CHU de Cocody, Abidjan, Côte d'Ivoire. \\ *Auteur correspondant; E-mail: kbaharrio@yahoo.fr, Tel: 00 (225) 0707303099
}

Received: 04-08-2021

Accepted: 23-12-2021

Published: 31-12-2021

\section{RESUME}

Les Indices de Voltage Ventriculaire Gauche (IVVG) électrocardiographiques sont reconnus pour avoir une faible sensibilité dans le diagnostic des anomalies de la masse ventriculaire gauche (MVG) comparativement à l'échocardiographie, méthode de référence, dont les mesures sont indexées à la Surface Corporelle (SC). Or, ces IVVG qui estiment la MVG à l'électrocardiographie ne sont pas en pratique indexés à la SC. La non-indexation des IVVG à la SC serait-elle à l'origine de leur faible sensibilité ? L'objectif de cette étude était de déterminer l'influence de la SC sur les IVVG dans une population de sujets sédentaires sains. Une étude expérimentale, prospective incluant 117 volontaires de 18 à 35 ans a été réalisée. Ils ont été reçus le matin à jeun. La prise du poids et de la taille ont servi au calcul de la SC. Un électrocardiogramme (ECG) standard a été réalisé au repos. L'amplitude des ondes $\mathrm{R}$ et $\mathrm{S}$ et la durée du complexe QRS ont été mesurées. Les indices de Sokolow-Lyon, R en aVL, de Cornell et le produit de Cornell ont été calculés puis indexés à la SC. Aucune corrélation significative n'a été obtenue entre les IVVG étudiés et la SC. L'indexation des IVVG à la SC n'a pas modifié la différence observée entre le groupe de sujets à valeurs normales non indexées et celui à valeurs élevées non indexées. Les IVVG n'ont pas été influencés par la SC dans cette étude. Une approche incluant à la fois la mesure de la SC et celle du tour de buste pourrait aider à la recherche sur l'amélioration de l'apport diagnostique des IVVG.

(C) 2021 International Formulae Group. All rights reserved.

Mots clés : Electrocardiogramme de repos, indice de voltage ventriculaire gauche, surface corporelle.

\section{Influence of body surface area on left ventricular voltage indices in healthy sedentary subjects}

\begin{abstract}
Electrocardiographic Left Ventricular Voltage Indices (LVVI) are known to have low sensitivity in the diagnosis of left ventricular mass (LVM) abnormalities compared with echocardiography, the reference method, whose measurements are indexed to body surface area (BSA). However, these LVTI that estimate
\end{abstract}


LVM on electrocardiography are not in practice indexed to BSA. Could the non-indexation of LVTI to BSA be the cause of their low sensitivity? The objective of this study was to determine the influence of BSA on LVVI in a population of healthy sedentary subjects. This was an experimental, prospective study involving 117 volunteers aged 18 to 35 years. They were received in the morning on an empty stomach. Weight and height measurements were used to calculate the BSA. A standard electrocardiogram was performed at rest. R and S wave amplitude and QRS duration were measured. Sokolow-Lyon, R in aVL, Cornell index, and Cornell product were calculated and indexed to BSA. No significant correlation was obtained between the studied LVVI and BSA. Indexation of LVVI to BSA did not alter the observed difference between the non-indexed normal and non-indexed high group. LVTI were not influenced by BSA. An approach that includes both BSA and torso circumference measurement could aid research on improving the diagnostic contribution of LVTI.

(C) 2021 International Formulae Group. All rights reserved.

Keywords: Resting electrocardiogram, left ventricular voltage index, body surface area.

\section{INTRODUCTION}

Depuis plus de cent ans, les indices de voltage ventriculaire gauche (IVVG) sont des critères électrocardiographiques utilisés pour évaluer la masse du ventricule gauche (Hancock et al., 2009). Ils sont déterminés sur un électrocardiogramme (ECG) de surface 12 à 18 dérivations. Ces IVVG sont calculés à partir de l'amplitude et de la durée des complexes QRS qui traduisent l'activité électrique des ventricules. Différents IVVG ont été établis à partir de complexes QRS mesurés dans une à trois des 18 dérivations électrocardiographiques. Ces dernières explorent le cœur dans différents plans. Quatre IVVG sont les plus utilisés (Williams et al., 2018). L'indice de Sokolow-Lyon, calculé à partir des dérivations électrocardiographiques V1 et V5, explore le cœur dans le plan horizontal. L'indice $\mathrm{R}$ dans la dérivation électrocardiographique aVL étudie le cœur dans le plan frontal. L'indice et le produit de Cornell, définis à partir des dérivations électrocardiographiques V3 et $\mathrm{aVL}$, permettent une analyse du cœur dans les plans frontal et horizontal. Quant à l'évaluation directe morphologique, elle se fait à l'échocardiographie qui est l'examen de référence (Lang et al., 2005 ; Cohen et al., 2017).

Pour optimiser le diagnostic des anomalies de la masse ventriculaire gauche, l'échocardiographie utilise l'indexation de l'épaisseur des parois et des diamètres ventriculaires gauches à la surface corporelle. Cette indexation découle de travaux qui avaient relevé une corrélation positive entre la surface corporelle et les diamètres ventriculaires gauches (Ristow et al, 2010 ; Pfaffenberger et al., 2013) et également entre la surface corporelle et la masse ventriculaire gauche à l'échocardiographie (Ilercil et al., 2001; Devereux et al., 2001). Ainsi en pratique, les valeurs de référence échocardiographiques des diamètres mesurés et de la masse calculée du ventricule gauche sont exprimées par mètre carré de surface corporelle (Lang et al., 2005 ; Cohen et al., 2017).

Pour le diagnostic des anomalies de la masse ventriculaire gauche, la sensibilité des IVVG est faible comparativement à celle des paramètres échocardiographiques, allant de 5 à 50\% selon les IVVG (Niakara et al., 2002 ; Schröder et al., 2015 ; Cournand et al., 2015 ; Taboulet, 2020). Aussi, l'ECG étant le premier examen à visée diagnostique des anomalies de la masse ventriculaire gauche, l'objectif général de ce travail était de déterminer l'influence de la surface corporelle sur les IVVG dans une population de sujets sédentaires sains.

\section{MATERIEL ET METHODES Approbation éthique}

Cette étude a été approuvée par le comité d'éthique du Centre Hospitalier et Universitaire Yopougon (Abidjan-Côte d'Ivoire) et a respecté les lignes directives de la déclaration d'Helsenki. La problématique, le but, les objectifs et le protocole de l'étude ont été expliqués aux sujets. Un 
consentement éclairé a été signé par chaque sujet participant à l'étude. Une autorisation écrite du chef de Laboratoire de Physiologie et d'Explorations Fonctionnelles (LPE) de l'Unité de Formation et de Recherche Sciences Médicales d'Abidjan (UFRSMA) a été obtenue. Les mesures barrières relatives au Covid-19 étaient respectées.

\section{Population d'étude}

La population cible était constituée d'étudiants volontaires de l'Université Félix Houphouët-Boigny (UFHB). Les critères d'inclusion ont concerné les sujets âgés de 18 à 35 ans, d'origine noire africaine, ayant un score de Ricci et Gagnon inférieur à 35 (sujets inactifs et peu actifs), un indice de masse corporelle (IMC) inférieure à 30 kilogrammes par mètre carré et, les femmes étant à la première phase de leur cycle menstruelle (caractérisée par un taux d'hormones ovariennes bas). N'ont pas été inclus, les sujets qui ont présenté un antécédent personnel de facteurs majeurs de risque cardiovasculaire: hypertension artérielle (HTA), diabète, dyslipidémie et tabagisme. Il est admis que ces facteurs représentent un risque cardio-vasculaires (Yessoufou et al., 2008 ; Ntchapda et al., 2010,; Attinsounon et al., 2020) et respiratoires (Tchissambou et al., 2020) important. Une symptomatologie ou pathologie cardio-vasculaire ou bronchopulmonaire, une hémoglobinopathie, une infection récente (moins d'une semaine) ou en cours, un traitement médicamenteux à visée cardio-vasculaire en cours, un antécédent familial de mort subite ou de cardiopathie génétique ont également été des motifs de non inclusion. Au cours du déroulement de l'étude, l'absence ou le refus du protocole par le sujet et la présence d'une anomalie à l'ECG à type d'un bloc de branche ou d'une hypertrophie ventriculaire droite ont été des motifs d'exclusion. La taille minimale de l'échantillon $(\mathrm{N})$ d'étude a été calculée à 47 sujets à partir de la formule des tailles $(\mathbf{N}=$ $\left.\left[\left(\mathbf{Z}_{\alpha}+\mathbf{Z}_{\beta}\right) / \mathbf{C}\right]^{2}+\mathbf{3}\right)$ basée sur la détection d'un coefficient de corrélation de 0,4 (Hulley et al., 2013). Sur la base des critères de sélection, parmi 122 volontaires, 117 dont 37 femmes ont constitué notre population d'étude.

\section{Protocole d'étude}

Il s'est agi d'une étude expérimentale, prospective, transversale à visée descriptive qui a eu pour cadre le LPE de l'UFRSMA logée à l'UFHB. Les sujets inclus ont été convoqués le matin à jeun au LPE, selon un programme de passage. Le protocole d'étude a été réalisé en 3 étapes successives. Un bref interrogatoire a recherché un stress majeur, un signe fonctionnel survenu dans les 48 heures et une prise de médicament dans les 72 heures avant la convocation. Le poids a été ensuite mesuré chez un sujet déchaussé et les poches vides. La taille a été mesurée chez un sujet les pieds joints, le dos au mur et la tête orientée dans le plan dit de Francfort (Delpierre et al., 2013). Après cette mesure, les sujets ont été installés dans une salle calme, climatisée (température entre 22 et $24^{\circ} \mathrm{C}$ ) pour la réalisation d'un ECG standard 12 dérivations, au format 3 pistes et en tracé de longueur moyenne. L'appareil utilisé était de marque Mindray BeneHeart R12. Les ECG ont été réalisés par deux techniciens spécialistes en explorations fonctionnelles cardio-respiratoires. Le sujet a été installé en décubitus dorsal, les yeux fermés, les paumes posées à plat sur le lit, les poignets et chevilles dégagés de tout accessoire en métal (bijoux et ceintures), les poches vides, le thorax nu. Les électrodes ont été positionnées selon les recommandations de l'AHA/ACC/HRS (Kligfield et al., 2007 ; Hancock et al., 2009). Après cinq minutes de repos, les tracés ont été enregistrés à une vitesse de $25 \mathrm{~mm} / \mathrm{s}$ et un voltage de 10 $\mathrm{mm} / \mathrm{mV}$ avec un filtre actif (Kligfield et al., 2007; Hancock et al., 2009). L'enregistrement a été fait en apnée pour éviter l'influence des mouvements de la cage thoracique sur l'amplitude des ondes électrocardiographiques. Chaque tracé comportait au moins cinq complexes QRS par dérivation. Deux lectures de chaque tracé ECG imprimé ont été faites par deux spécialistes en cardiologie. Une troisième 
lecture a été effectuée par un troisième cardiologue lorsqu'il existait une différence de mesure de plus d'un millimètre en amplitude et de plus de 0,002 seconde en durée, entre les deux premières lectures.

\section{Paramètres étudiés}

La population d'étude a été répartie selon le genre. La surface corporelle (SC) a été calculée à partir du poids en kilogramme et de la taille en mètre grâce à la formule de Boyd : $\mathrm{SC}=0,0003207 \mathrm{x}$ Poids (0,7285$0,0188 \times \log ($ Poids) $)) \times\left(\right.$ Taille $\left.^{0,3}\right)$. L'IMC a été calculé grâce à la formule IMC = Poids/(Taille) ${ }^{2}$ en kg/m².

Dans la population globale, féminine et masculine, les moyennes de l'âge, de l'IMC et de la surface corporelle ont été calculées et comparées.

A l'ECG, le complexe QRS, signal électrique traduisant l'activation des ventricules a été étudié. Par convention, la première déflexion négative est appelée onde $\mathrm{Q}$, la première déflexion positive onde $\mathrm{R}$, la négativité suivant l'onde $\mathrm{R}$, est appelée onde S (Taboulet, 2021). Les amplitudes des ondes $\mathrm{R}$ et $\mathrm{S}$ ont été mesurées dans toutes les dérivations, sauf en aVR, de la ligne de base au sommet de l'onde. Dans une dérivation, la valeur de l'amplitude de l'onde $\mathrm{R}$ retenue a été la moyenne des amplitudes de toutes les ondes $\mathrm{R}$ de ladite dérivation. Il en a été de même pour les ondes $\mathrm{S}$ dans chaque dérivation. La durée du complexe QRS a été déterminée dans la dérivation V5, du début de la $1^{\text {ère }}$ déflexion de la ligne isoélectrique survenant après l'onde $\mathrm{P}$ jusqu'au point $\mathrm{J}$. La durée retenue du complexe QRS a été la moyenne des durées des QRS de ladite dérivation.

Pour ce travail, les quatre IVVG les plus utilisés ont été calculés à partir des amplitudes des ondes $\mathrm{R}$ et $\mathrm{S}$ et de la durée du complexe QRS de dérivation selon l'ESC (Williams et al., 2018). L'indice de SokolowLyon (SV1 + RV5) a été dit normal pour une valeur inférieure à 45 millimètres $(\mathrm{mm})$ chez le sujet ayant un âge inférieur à 40 ans (Taboulet, 2020). L'indice $\mathrm{R}$ en aVL (amplitude de l'onde $\mathrm{R}$ dans la dérivation aVL) a été noté normal si la valeur est inférieure à $11 \mathrm{~mm}$. L'indice de Cornell $(\mathrm{RaVL}+\mathrm{SV} 3)$ dont la valeur normale a été inférieure ou égale à $20 \mathrm{~mm}$ chez la femme et inférieure ou égale à $28 \mathrm{~mm}$ chez l'homme. Le produit de Cornell [(RaVL + SV3 $) x$ durée QRS] dont la valeur normale a été inférieure ou égale à $2440 \mathrm{~mm} . \mathrm{ms}$. Chez chaque sujet, chacun des quatre IVVG a été indexé à la surface corporelle. Les moyennes de chaque IVVG (non indexé et indexé) ont été établies dans la population d'étude dans chaque genre à partir des valeurs individuelles. Ces moyennes ont été comparées entre elles.

Une corrélation a été recherchée entre chaque IVVG et la surface corporelle dans la population d'étude. Pour chaque indice, les sujets ont été classés en deux groupes : ceux ayant un indice normal et ceux ayant un indice élevé. Dans chaque groupe, les valeurs moyennes de l'indice (non indexées et indexées) ont été déterminées. Les indices normaux et élevés ont été comparés avant et après indexation à la surface corporelle.

\section{Analyse statistique}

Les analyses statistiques ont été effectuées grâce au logiciel $\mathrm{R}$ 4.0.2. Les moyennes ont été comparées grâce aux tests de Wilcoxon-Mann-Whitney. Les données catégorielles ont été décrites par leur effectif et pourcentage. Les indices ont été catégorisés en deux classes «normale » et «élevée » afin d'être analysés comme des données catégorielles. Des régressions linéaires multiples et logistiques ont été utilisées pour décrire l'influence des variables sur les indices respectivement en valeur continue et en classes d'indice. Le seuil d'erreur a été fixé à 0,05 .

\section{RESULTATS}

\section{Age et caractéristiques anthropométriques}

Les caractéristiques anthropométriques et électrocardiographiques de la population d'étude ont été répertoriés dans le Tableau 1. L'âge et l'IMC des femmes n'ont pas été 
statistiquement différents de celui des hommes avec une surface corporelle statistiquement plus petite. Pour l'indice de Sokolow-Lyon, les indices et produit de Cornell, les moyennes non indexées et indexées, ont été significativement plus élevées chez les hommes comparativement aux femmes. L'indice $\mathrm{R}$ en aVL non indexé et indexé, a été statistiquement similaire entre hommes et femmes. Après ajustement de l'âge, du genre et de l'indice de masse corporelle, l'augmentation de la surface corporelle a entrainé une baisse non significative de l'indice de Sokolow Lyon non indexé de 39\% ( $\mathrm{p}=0,08)$. Pour l'indice $\mathrm{R}$ en aVL, les indices et produit de Cornell, il n'a pas été trouvé de relation linéaire directe avec la surface corporelle.
Dans la population globale et le groupe des hommes, l'âge et la surface corporelle ont été statistiquement similaires entre les sujets ayant un indice de Sokolow-Lyon (Tableau 2) et un indice de Cornell (Tableau 3) normal et ceux ayant un indice de Sokolow-Lyon et indice de Cornell élevé. Aucune femme n'a eu un indice de Sokolow-Lyon et indice de Cornell élevés. Dans la population globale et le groupe des hommes, la différence statistiquement significative observée entre les indices de Sokolow-Lyon (Tableau 2) et les indices de Cornell (Tableau 3) normaux et élevés non indexés s'est maintenue après avoir indexé ces indices normaux et élevés à la surface corporelle. Dans la population globale, aucun sujet n'a eu un indice $\mathrm{R}$ en aVL et un produit de Cornell élevé.

Tableau 1 : Caractéristiques anthropométriques et électrocardiographiques de la population d'étude.

\begin{tabular}{|c|c|c|c|c|}
\hline PARAMETRES & $\begin{array}{c}\text { TOUS } \\
(n=117)\end{array}$ & $\begin{array}{c}\text { FEMMES } \\
(n=37)\end{array}$ & $\begin{array}{c}\text { HOMMES } \\
(n=80)\end{array}$ & $\mathbf{p}$ \\
\hline \multicolumn{5}{|l|}{ Anthropométriques } \\
\hline Age (ans) & $22,7 \pm 3,0$ & $22,3 \pm 2,7$ & $22,8 \pm 3,1$ & 0,36 \\
\hline $\operatorname{IMC}\left(\mathrm{kg} / \mathrm{m}^{2}\right)$ & $21,8 \pm 2,6$ & $22,1 \pm 3,3$ & $21,7 \pm 2,2$ & 0,60 \\
\hline Surface corporelle $\left(\mathbf{m}^{2}\right)$ & $1,72 \pm 0,14$ & $1,63 \pm 0,14$ & $1,76 \pm 0,12$ & $<0,001$ \\
\hline \multicolumn{5}{|l|}{ Electrocardiographiques } \\
\hline \multicolumn{5}{|l|}{ Indice de Sokolow-Lyon } \\
\hline Non indexé (mm) & $31,3 \pm 9,9$ & $24,1 \pm 6,4$ & $34,7 \pm 9,5$ & $<0,001$ \\
\hline Indexé $\left(\mathrm{mm} / \mathrm{m}^{2}\right)$ & $18,3 \pm 5,9$ & $14,8 \pm 4,2$ & $19,9 \pm 5,9$ & $<0,001$ \\
\hline \multicolumn{5}{|l|}{ Indice $\mathrm{R}$ en aVL } \\
\hline Non indexé (mm) & $3,3 \pm 2,5$ & $2,8 \pm 1,6$ & $3,5 \pm 2,7$ & 0,29 \\
\hline Indexé $\left(\mathrm{mm} / \mathrm{m}^{2}\right)$ & $1,9 \pm 1,4$ & $1,7 \pm 1,0$ & $2,0 \pm 1,6$ & 0,65 \\
\hline \multicolumn{5}{|l|}{ Indice de Cornell } \\
\hline Non indexé (mm) & $15,4 \pm 8,9$ & $8,8 \pm 4,0$ & $18,4 \pm 8,9$ & $<0,001$ \\
\hline Indexé $\left(\mathrm{mm} / \mathrm{m}^{2}\right)$ & $8,9 \pm 5,2$ & $5,43 \pm 2,6$ & $10,6 \pm 5,3$ & $<0,001$ \\
\hline \multicolumn{5}{|l|}{ Produit de Cornell } \\
\hline Non indexé (mm) & $1349,6 \pm 797,5$ & $723,8 \pm 335,5$ & $1639,1 \pm 783,8$ & $<0,001$ \\
\hline Indexé $\left(\mathrm{mm} / \mathrm{m}^{2}\right)$ & $784,2 \pm 460,9$ & $447,4 \pm 215,8$ & $940,0 \pm 462,0$ & $<0,001$ \\
\hline
\end{tabular}


Tableau 2: Indice de Sokolow Lyon selon la valeur normale ou élevée de l'indice dans la population d'étude et selon le genre.

\begin{tabular}{|c|c|c|c|c|c|c|c|}
\hline \multirow[t]{3}{*}{ POPULATIONS } & \multirow[t]{3}{*}{ PARAMETRES } & \multicolumn{6}{|c|}{ INDICE DE SOKOLOW-LYON } \\
\hline & & $\begin{array}{c}\text { Valeurs } \\
\text { normales } \\
\quad(<45 \\
\mathrm{mm})\end{array}$ & $\begin{array}{c}\text { Valeurs } \\
\text { élevées } \\
(\geq 45 \\
\mathrm{mm})\end{array}$ & & $\begin{array}{c}\text { Valeurs } \\
\text { normales } \\
(<45 \\
\mathrm{mm})\end{array}$ & $\begin{array}{c}\text { Valeurs } \\
\text { élevées } \\
(\geq 45 \\
\mathrm{mm})\end{array}$ & \\
\hline & & \multicolumn{2}{|c|}{ Non indexées } & $\mathbf{p}$ & \multicolumn{2}{|c|}{ Indexées } & $\mathbf{p}$ \\
\hline \multirow{4}{*}{$\begin{array}{l}\text { TOUS } \\
(\mathrm{n} 1=107 ; \mathbf{n} 2= \\
10)\end{array}$} & $\begin{array}{l}\text { Indice }(\mathrm{mm} ; \\
\left.\mathrm{mm} / \mathrm{m}^{2}\right)\end{array}$ & $\begin{array}{c}29,40 \pm \\
7,1\end{array}$ & $\begin{array}{c}51,7 \pm \\
7,6\end{array}$ & $<0,001$ & $\begin{array}{c}17,2 \pm \\
4,6\end{array}$ & $\begin{array}{c}30,1 \pm \\
4,6\end{array}$ & $<0,001$ \\
\hline & Age (année) & $\begin{array}{c}22,7 \pm \\
3,0\end{array}$ & $\begin{array}{c}22,2 \pm \\
2,8\end{array}$ & 0,81 & - & - & - \\
\hline & $\operatorname{IMC}\left(\mathrm{kg} / \mathrm{m}^{2}\right)$ & $\begin{array}{c}21,9 \pm \\
2,6\end{array}$ & $\begin{array}{c}21,3 \pm \\
2,0\end{array}$ & 0,46 & - & - & - \\
\hline & $\mathrm{SC}\left(\mathrm{m}^{2}\right)$ & $\begin{array}{c}1,72 \pm \\
0,14\end{array}$ & $\begin{array}{c}1,72 \pm \\
0,11\end{array}$ & 1,00 & - & - & \\
\hline \multirow[t]{4}{*}{$\begin{array}{l}\text { FEMMES } \\
(\mathbf{n} 1=37 ; \mathbf{n} 2=0)\end{array}$} & $\begin{array}{l}\text { Indice }(\mathrm{mm} ; \\
\left.\mathrm{mm} / \mathrm{m}^{2}\right)\end{array}$ & $\begin{array}{c}24,1 \pm \\
6,4\end{array}$ & - & - & $\begin{array}{c}14,8 \pm \\
4,2\end{array}$ & - & - \\
\hline & Age (année) & $\begin{array}{c}22,2 \pm \\
2,7\end{array}$ & - & - & & & - \\
\hline & $\operatorname{IMC}\left(\mathrm{kg} / \mathrm{m}^{2}\right)$ & $\begin{array}{c}22,1 \pm \\
3,3\end{array}$ & - & - & & & - \\
\hline & $\mathrm{SC}\left(\mathrm{m}^{2}\right)$ & $\begin{array}{c}1,63 \pm \\
0,14\end{array}$ & - & - & & & - \\
\hline \multirow[t]{4}{*}{$\begin{array}{l}\text { HOMMES } \\
(\mathrm{n} 1=70 ; \mathrm{n} 2=10)\end{array}$} & $\begin{array}{l}\text { Indice }(\mathrm{mm} ; \\
\left.\mathrm{mm} / \mathrm{m}^{2}\right)\end{array}$ & $\begin{array}{c}32,2 \pm \\
6,8\end{array}$ & $\begin{array}{c}51,7 \pm \\
7,6\end{array}$ & $<0,001$ & $\begin{array}{c}18,4 \pm \\
4,4\end{array}$ & $\begin{array}{c}30,1 \pm \\
4,6\end{array}$ & $<0,001$ \\
\hline & Age (année) & $\begin{array}{c}23,0 \pm \\
3,1\end{array}$ & $\begin{array}{c}22,2 \pm \\
2,8\end{array}$ & 0,65 & - & - & \\
\hline & $\mathrm{IMC}\left(\mathrm{kg} / \mathrm{m}^{2}\right)$ & $\begin{array}{c}21,7 \pm \\
2,2\end{array}$ & $\begin{array}{c}21,3 \pm \\
2,0\end{array}$ & 0,43 & - & - & \\
\hline & $\mathrm{SC}\left(\mathrm{m}^{2}\right)$ & $\begin{array}{c}1,76 \pm \\
0,13\end{array}$ & $\begin{array}{c}1,72 \pm \\
0,12\end{array}$ & 0,31 & - & - & \\
\hline
\end{tabular}

Les données sont exprimées en moyenne \pm écart-type ; indice non indexé en $\mathrm{mm} ;$ indice indexé en $\mathrm{mm} / \mathrm{m}^{2} ; \mathrm{n}$ : effectif ; $\mathrm{n}_{1}=$ effectif pour indice normal ; $\mathrm{n}_{2}=$ effectif pour indice élevé ; IMC : indice de masse corporelle ; SC : surface corporelle ; indice de Sokolow-Lyon indexé : $\left(\mathrm{SV}_{1}+\mathrm{RV}_{5}\right) / \mathrm{SC} ; \mathrm{p}$ : test t de significativité (p significatif pour une valeur $\left.<0,05\right)$. 
Tableau 3 : Indice de Cornell selon la valeur normale ou élevée de l'indice dans la population d'étude et selon le genre.

\begin{tabular}{|c|c|c|c|c|c|}
\hline \multirow[t]{7}{*}{ POPULATIONS } & \multirow[t]{7}{*}{ PARAMETRES } & \multicolumn{4}{|c|}{ INDICE DE CORNELL } \\
\hline & & $\begin{array}{l}\text { Valeurs } \\
\text { normales }\end{array}$ & $\begin{array}{l}\text { Valeurs } \\
\text { élevées }\end{array}$ & $\begin{array}{c}\text { Valeurs } \\
\text { normales }\end{array}$ & $\begin{array}{l}\text { Valeurs } \\
\text { élevées }\end{array}$ \\
\hline & & $\leq 20 \mathrm{~mm}$ & (femmes & $\leq 20 \mathrm{~mm}$ & (femmes \\
\hline & & hommes & $>20$ & hommes & $>20$ \\
\hline & & $\leq 28 \mathrm{~mm})$ & $\mathrm{mm}$ & $\leq 28 \mathrm{~mm})$ & $\mathrm{mm}$, \\
\hline & & & hommes & & hommes \\
\hline & & & $\begin{array}{l}>28 \\
\mathrm{~mm})\end{array}$ & & $\begin{array}{l}>28 \\
\mathrm{~mm})\end{array}$ \\
\hline
\end{tabular}

\begin{tabular}{|c|c|c|c|c|c|c|c|}
\hline \multirow{3}{*}{$\begin{array}{l}\text { TOUS } \\
(\mathrm{n} 1=107 ; \mathrm{n} 2= \\
10)\end{array}$} & \multirow[b]{2}{*}{$\begin{array}{l}\text { Indice }(\mathrm{mm} ; \\
\left.\mathrm{mm} / \mathrm{m}^{2}\right)\end{array}$} & \multicolumn{2}{|c|}{ Non indexées } & \multirow{2}{*}{$\frac{\mathbf{p}}{<0,001}$} & \multicolumn{2}{|c|}{ Indexées } & \multirow{2}{*}{$\frac{\mathbf{p}}{<0,001}$} \\
\hline & & $\begin{array}{c}13,1 \pm \\
6,0\end{array}$ & $\begin{array}{c}35,0 \pm \\
5,2\end{array}$ & & $7,1 \pm 3,5$ & $\begin{array}{c}20,1 \pm \\
3,6\end{array}$ & \\
\hline & Age (année) & $\begin{array}{c}22,7 \pm \\
3,0\end{array}$ & $\begin{array}{c}22,0 \pm \\
2,6\end{array}$ & 0,35 & - & - & - \\
\hline & $\operatorname{IMC}\left(\mathrm{kg} / \mathrm{m}^{2}\right)$ & $\begin{array}{c}21,9 \pm \\
2,6\end{array}$ & $\begin{array}{c}21,3 \pm \\
2,6\end{array}$ & 0,48 & - & - & - \\
\hline & $\mathrm{SC}\left(\mathrm{m}^{2}\right)$ & $\begin{array}{c}1,71 \pm \\
0,14\end{array}$ & $\begin{array}{c}1,75 \pm \\
0,15\end{array}$ & 0,71 & - & - & \\
\hline \multirow[t]{4}{*}{$\begin{array}{l}\text { FEMMES } \\
(\mathrm{n} 1=37 ; \mathrm{n} 2=0)\end{array}$} & $\begin{array}{l}\text { Indice (mm; } \\
\left.\mathrm{mm} / \mathrm{m}^{2}\right)\end{array}$ & $8,8 \pm 4,0$ & - & - & $5,4 \pm 2,6$ & - & - \\
\hline & Age (année) & $\begin{array}{c}22,2 \pm \\
2,7\end{array}$ & - & - & & & - \\
\hline & $\operatorname{IMC}\left(\mathrm{kg} / \mathrm{m}^{2}\right)$ & $\begin{array}{c}22,1 \pm \\
3,3\end{array}$ & - & - & & & - \\
\hline & $\mathrm{SC}\left(\mathrm{m}^{2}\right)$ & $\begin{array}{c}1,63 \pm \\
0,14\end{array}$ & - & - & & & - \\
\hline \multirow[t]{4}{*}{$\begin{array}{l}\text { HOMMES } \\
(\mathrm{n} 1=68 ; \mathrm{n} 2=12)\end{array}$} & $\begin{array}{l}\text { Indice (mm; } \\
\left.\mathrm{mm} / \mathrm{m}^{2}\right)\end{array}$ & $\begin{array}{c}15,5 \pm \\
5,6\end{array}$ & $\begin{array}{c}35,0 \pm \\
5,2\end{array}$ & $<0,001$ & $8,9 \pm 3,3$ & $\begin{array}{c}20,1 \pm \\
3,6\end{array}$ & $<0,001$ \\
\hline & Age (année) & $\begin{array}{c}23,0 \pm \\
3,2\end{array}$ & $\begin{array}{c}22,0 \pm \\
2,6\end{array}$ & 0,24 & - & - & \\
\hline & $\operatorname{IMC}\left(\mathrm{kg} / \mathrm{m}^{2}\right)$ & $\begin{array}{c}21,7 \pm \\
2,1\end{array}$ & $\begin{array}{c}21,3 \pm \\
2,6\end{array}$ & 0,51 & - & - & \\
\hline & $\mathrm{SC}\left(\mathrm{m}^{2}\right)$ & $\begin{array}{c}1,76 \pm \\
0,1\end{array}$ & $\begin{array}{c}1,75 \pm \\
0,15\end{array}$ & 0,47 & - & - & \\
\hline
\end{tabular}

Les données sont exprimées en moyenne \pm écart-type $; \mathrm{n}$ : effectif $; \mathrm{n} 1=$ effectif pour indice normal $; \mathrm{n} 2=$ effectif pour indice élevé ; IMC : indice de masse corporelle ; SC : surface corporelle ; indice de Cornell indexé : (SV3+RaVL) /SC ; p : test t de significativité ( $\mathrm{p}$ significatif pour une valeur $<0,05$. 


\section{DISCUSSION}

Le but de ce travail a été de déterminer l'influence de la surface corporelle sur les quatre IVVG les plus utilisés à l'ECG, dans une population de sujets sains et sédentaires. Notre étude n'a pas retrouvé de corrélation entre la surface corporelle et ces quatre IVVG. Dans les populations globale, masculine et féminine, l'indexation des IVVG à la surface corporelle (indices de Sokolow-Lyon et de Cornell) n'a pas modifié la différence observée entre le groupe de sujets à valeurs normales non indexées et celui à valeurs élevées non indexées.

Les données de la littérature ont relevé une corrélation positive entre d'une part la surface corporelle et d'autre part la masse ventriculaire du cœur au plan anatomique (Xue et al., 2019; Zhang et al., 2019) et au plan échocardiographique (référence pour la mesure directe de la masse ventriculaire gauche) (Lang et al., 2005 ; Ristow et al., 2010 ; Pfaffenberger et al., 2013 ; Cohen et al., 2017 ; Ahmed et al., 2020). Le calcul des IVVG représente une approche indirecte de mesure de la masse ventriculaire gauche à l'ECG.

L'indice de Sokolow-Lyon et l'indice $\mathrm{R}$ en aVL explorent la masse musculaire du ventricule gauche uniquement dans un seul plan, respectivement horizontal et frontal (Taboulet, 2020). Ces IVVG ont été proposés en 1949 pour le diagnostic de l'hypertrophie ventriculaire gauche électrique (Hancock et al., 2009). Leur sensibilité est faible, respectivement de 5\% (Schröder et al., 2015) et proche de 20\% (Courand et al., 2015). En vue d'améliorer la performance et la précision diagnostique des anomalies de la masse ventriculaire gauche, l'indice de Cornell a été proposé (Taboulet, 2020). Cet indice explore le cour dans les plans horizontal et frontal et sa sensibilité serait de 30-40\% (Niakara et al., 2002 ; Taboulet, 2020). D'autres travaux ont insisté sur la nécessité d'aller au-delà des simples mesures d'amplitude de complexe QRS pour améliorer la performance de l'ECG dans la détection des anomalies de la masse ventriculaire gauche (Courand et al., 2015; Taboulet, 2020). L'une des approches a été d'incorporer aux amplitudes du complexe QRS la durée de ce complexe QRS (Courand et al., 2015 ; Taboulet, 2020). La durée du complexe QRS est un paramètre indispensable pour apprécier la conduction intraventriculaire et identifier une augmentation de la masse ventriculaire. Elle varie proportionnellement avec la masse du ventricule gauche (Taboulet, 2020). La sensibilité du produit de Cornell combinée à l'indice de Sokolow-Lyon serait entre 45 et $50 \%$ (Wachtell et al., 2001).

Dans notre travail, l'absence de corrélation entre la surface corporelle et les IVVG pourrait s'expliquer par le fait qu'en raison de leur caractère indirect dans l'estimation de la masse ventriculaire, les mesures ECG seraient influencées par divers facteurs dont les mouvements respiratoires et la position du cour dans le médiastin (Guyton et al., 2003 ; Chapelon-Abric, 2004 ; Sende, 2009 ; Swenson et al., 2011).

$\mathrm{La}$ respiration normale s'accompagnerait de variations cycliques de l'amplitude des QRS : l'inspiration profonde réduirait cette amplitude (Chapelon-Abric, 2004). La position du cœur dans le médiastin influencerait les amplitudes des ondes $\mathrm{R}$ et $\mathrm{S}$ (Guyton et al., 2003 ; Sende, 2009 ; Swenson et al., 2011).

Contrairement aux indices de Sokolow-Lyon et $\mathrm{R}$ en aVL l'indice et le produit de Cornell explorent la masse du ventricule gauche simultanément dans les deux plans frontal et horizontal (Taboulet, 2020). Ces indices ont une forte corrélation échocardiographique avec la masse ventriculaire gauche indexée à la surface corporelle (Lang et al., 2005 ; Ristow et al, 2010 ; Pfaffenberger et al., 2013 ; Cohen et al., 2017 ; Ahmed et al., 2020). Cependant, tout comme l'indice de Sokolow-Lyon et l'indice $\mathrm{R}$ en aVL, l'indice et le produit de Cornell demeurent une approche indirecte de mesure de la masse ventriculaire gauche qui pourrait être influencés par l'amplitude de la respiration et la position du cœur dans le médiastin (Swenson et al., 2011). Dans cette 
étude, les ECG ont été réalisés en apnée pour éliminer l'influence des mouvements respiratoires. Les mesures du tour du buste et de la composition corporelle pour évaluer la masse grasse permettrait de déterminer le rôle de ces facteurs dans le calcul des IVVG.

\section{Conclusion}

Cette étude avait pour but de rechercher l'influence de la surface corporelle sur les IVVG. Dans notre population de sujets noirs africains sédentaires sains, aucune relation statistiquement significative n'a été mise en évidence entre la surface corporelle et les indices de Sokolow-Lyon, R aVL, de Cornell et le produit de Cornell. L'indexation de ces IVVG à la surface corporelle n'a pas permis d'améliorer la performance diagnostique des anomalies de la masse ventriculaire gauche. Une approche incluant à la fois la mesure de la surface corporelle et celle du tour de buste et l'utilisation d'autres IVVG pourrait aider à la recherche sur l'amélioration de l'apport diagnostique des IVVG.

\section{CONFLITS D'INTERETS}

Les auteurs déclarent ne pas avoir de conflits d'intérêts en relation avec cet article.

\section{CONTRIBUTIONS DES AUTEURS}

KBA et DTK ont rédigé le protocole de l'étude. BSE a validé le protocole. KBA, DTK, KBM, KL, BKJ, AA et YPD ont participé à la collecte des données. KBA et BSE ont écrit l'article. Tous les co-auteurs ont contribué à la relecture et à la finalisation du manuscrit.

\section{REMERCIEMENTS}

Nous remercions tous les étudiants de l'UFHB qui ont accepté de participer à cette étude et tout le personnel du LPE de l'UFRSMA.

\section{REFERENCES}

Ahmed SN, Jhaj R, Sadasivam B, Joshi R. 2020. Prediction of Left Ventricular Mass Index Using Electrocardiography in Essential Hypertension - A Multiple Linear Regression Model. Medical Devices: Evidence and Research, 13: 163-72. DOI: 10.2147/mder.s253792.

Attinsounon CA, Codjo LH, Ahouansou LM, Dohou SHM, Alassani A et Houenassi MD. 2020. Risque cardiovasculaire chez les adultes vivant avec le virus d'immunodéficience humaine et sous traitement antirétroviral à Parakou au Bénin. Int. J. Biol. Chem. Sci., 14(1): 213-24.

DOI: https://dx.doi.org/10.4314/ijbcs.v14i1.18

Chapelon-Abric C. 2004. Méthode d'Analyse des Electrocardiogrammes de Surface Douze Dérivations. Encyclopédie Médico-Chirurgicale.

Cohen A, Soulat-Dufour L, Ancedy Y, Bedet A, Chauvet M, Cohen S, Ederhy S, El Asri C, Etienney, Goublaire C, Labbé V. 2017. Echocardiographie en Pratique. Lavoisier; p437-41.

Courand PY, Grandjean A, Charles P, Paget V, Khettab F, Bricca G, Boussel L, Lantelme P, Harbaoui B. 2015. R Wave in aVL Lead Is a Robust Index of Left Ventricular Hypertrophy: A Cardiac MRI Study. Am Journ Hypertens; 28 (8): 1038-48. DOI :10.1093/ajh/hpu268

Delpierre S, Badier M, Guillot C, Monnier A. 2013. Vademecum d'Explorations Fonctionnelles Respiratoires (1 $1^{\mathrm{èr}}$ édn). De Boeck : Bruxelles ; p. 24.

Devereux RB, Bella JN, Palmieri V Oberman A, Kitzman DW, Hopkins PN, Rao DC, Morgan D, Paranicas M, Fishman D, Arnett DK . 2001. Left ventricular systolic dysfunction in a biracial sample of hypertensive adults: the hypertension genetic epidemiology network (HyperGEN) study. Hypertension, 38: 417-23. DOI : 10.1161/01.hyp.38.3.417.

Guyton AC, Hall JE. 2003. Précis de Physiologie Médicale ( $2^{\mathrm{e}}$ édn). Piccin Nuova Libraria : Padoue; p. 147.

Hancock EW, Deal BJ, Mirvis DM Okin P, Kligfield P, Gettes LS. 2009. AHA/ACCF/HRS Recommendations for the Standardization and Interpretation of 
the Electrocardiogram Part V: Electrocardiogram Changes Associated With Cardiac Chamber Hypertrophy. Circulation; 119: 251-61. DOI: 10.1161/circulationaha.108.191097.

Hulley SB, Cummings SR, Browner WS, Grady D, Newman TB. 2013. Designing Clinical Research: an Epidemiologic Approach (4th edn). Lippincott Williams \& Wilkins : Philadelphia, PA; 79p.

Ilercil A, O'Grady MJ, Roman MJ Paranicas M, Lee ET, Welty TK, Fabsitz RR, Howard BV, Devereux RB. 2001. Reference values for echocardiographic measurements in urban and rural populations of differing ethnicity: the strong heart study. $J$ Am Soc Echocardiogr, 14: 601-11. DOI: 10.1067/mje.2001.113258.

Kligfield P, Gettes LS, Bailey JJ Bailey JJ, Childers R, Deal JB, Hancock EW, Herpen GV, Kors JA, Macfarlane P, Mirvis DM, Pahlm O, Rautaharju P, Wagner GS. 2007. Recommendations for the Standardization and Interpretation of the Electrocardiogram. Part I: The Electrocardiogram and Its Technology. Circulation; 115: 1306-24. DOI: 10.1161/circulationaha.106.180200.

Lang RM, Bierig M, Devereux RB. 2005. ASE committee recommendations. Recommandations pour la Quantification des Cavités Cardiaques. $J$ Am Soc Echocardiogr, 18: 1440-63. DOI: 10.1016/j.echo.2005.10.005.

Niakara A, Ouédraogo N, Nébié LVA, Kaboré NJP, Megnigbeto CA. 2002. Indices électrocardiographiques de routine pour le diagnostic de l'hypertrophie ventriculaire gauche: performances chez le sujet noir africainRoutine electrocardiographic criteria for the diagnosis of left ventricular hypertrophy : Performance in Black African. Ann Cardiol Angeio, 51(4) : 193-198. DOI: https://doi.org/10.1016/S0003-

3928(02)00096-3Get rights and content.

Ntchapda F, Dimo T, Atchade AT. 2010. Antihypertensive effects of the methylene chloride leaf extract of Celtis durandii Engler (Ulmaceae) on rats. Int. J. Biol. Chem. Sci., 4(3): 642-48. DOI: 10.4314/ijbcs.v4i3.60471

Pfaffenberger S, Bartko P, Graf A, Pernicka E, Babayev J, Lolic E, Bonderman D, Baumgartner H, Maurer G, Mascherbauer J. 2013. Size Matters! Impact of Age, Sex, Height, and Weight on the Normal Heart Size. Circ Cardiovasc Imaging, 6: 1073-9. DOI: 10.1161/circimaging.113.000690.

Ristow B, Ali S, Na B, Turakhia PM, Whooley, Schiller NB. 2010. Predicting Heart Failure Hospitalization and Mortality by Quantitative Echocardiography: Is Body Surface Area the Indexing Method of Choice? The Heart and Soul Study. J Am Soc Echocardiogr, 23(4): 406-13. DOI: 10.1016/j.echo.2010.01.019.

Sende J. 2009. Guide Pratique de l'ECG (2 édn). Estem : Paris ; p. 80.

Schröder J, Nuding S, Müller-Werdan U, Werdan K, Kluttig A, Russ M, Greiser KH, Kors JA, Haerting J, Medenwald D. 2015. Performance of Sokolow-Lyon index in detection of echocardiographically diagnosed left ventricular hypertrophy in a normal Eastern German population - results of the CARLA study. BMC Cardiovasc Disord, 15(1): 69-75. DOI: 10.1186/s12872-015-0066-5.

Swenson DJ, Geneser SE, Stinstra JG, Kirby RM, MacLeod RS. 2011. Cardiac Position Sensitivity Study in the Electrocardiographic Forward Problem Using Stochastic Collocation and Boundary Element Methods. Ann Biomed Eng., 39(12): 2900-2910. DOI: 10.1007/s10439-011-0391-5.

Taboulet P. 2020. https://www.ecardiogram.com/indice-dhvg-1generalites/ accès le 16 avril 2021 à 07 h gmt.

Taboulet P. 2020. https://www.ecardiogram.com/indice-dhvg-3-cornell/ accès le 10 décembre 2021 à 23 h gmt. 
Taboulet P. 2021. https://www.ecardiogram.com/complexe-qrs/ accès le 10 décembre 2021 à $00 \mathrm{~h}$ gmt.

Tchissambou BP, Massamba A, Mabiala JR et Senga P. 2010. Effets du tabagisme et du niveau de dépendance nicotinique sur les métabolismes anaérobies des footballeurs. Int. J. Biol. Chem. Sci., 4(6): 2082-2092.

Wachtell K, Rokkedal J, Bella JN, Aalto T, Dahlöf B, Smith G, et al. 2001. Effect of electrocardiographic left ventricular hypertrophy on left ventricular systolic function in systemic hypertension (The LIFE Study). Losartan Intervention for Endpoint. Am. J. Cardiol, 87(1): 54-60. DOI: https://doi.org/10.101016/S0002914(00)01272-8.

Williams B, Mancia G, Spiering W, Rosei EA, Azizi M, Burnier M, Clement DL, Coca A, de Simone G, Dominiczak A, Kahan T, Mahfoud F, Redon, Ruilope L, Zanchetti A, Kerins M, Kjeldsen SE, Kreutz R, Laurent S, Lip GYH, McManus R, Narkiewicz K, Ruschitzka F, Schmieder RE, Shlyakhto E, Tsioufis C, Aboyans V, Desormais I. 2018. 2018
ESC/ESH Guidelines for themanagement of arterial hypertension. Eur Heart J, 39(33): 3021-104. DOI: 10.1093/eurheartj/ehy339.

Xue JJ, Wang TQ, Jia YQ, Xiao Y, Tian MH, D W Guan DW, Zhang GH, Wu $\mathrm{X}$, Li RB, Zhao R, Cao ZP, Zhu BL. 2019. Statistical Analysis of the Heart and Lung Mass. Forensic Anatomical Cases and Its Forensic Significance, 35(6): 651-6. DOI: 10.12116/j.issn.10045619.2019.06.002.

Yessoufou A, Atègbo JM, Moutairou K, Dramane KL et Khan NA. 2008. Malnutrition-modulated diabetes mellitus (MMDM) : a state of review. Int. J. Biol. Chem. Sci., 2(2): 230-238.

Zhang W, Zhou Y, Bai B, Yu S, Xiong J, Chi C, Teliewubai J, Li Jue, Blacher J, Zhang Y, Xu Y. 2019. Consistency of left ventricular hypertrophy diagnosed by electrocardiography and echocardiography: the Northern Shanghai Study. Clin Interv Aging, 11(14): $\quad 549-556 . \quad$ DOI: http://dx.org/10.2147/CI.S180723 